\title{
Numerical spurious solutions in the effective mass approximation
}

X. Cartoixà

T. J. Watson, Sr, Laboratories of Applied Physics 128-95, California Institute of Technology, Pasadena, California 91125

D. Z.-Y. Ting

Jet Propulsion Laboratory, California Institute of Technology, Pasadena, California 91109

T. C. McGilla)

T. J. Watson, Sr., Laboratories of Applied Physics 128-95, California Institute of Technology, Pasadena, California 91125

(Received 4 November 2002; accepted 26 December 2002)

\begin{abstract}
We have characterized a class of spurious solutions that appears when using the finite difference method to solve the effective mass approximation equations. We find that the behavior of these solutions as predicted by our model shows excellent agreement with numerical results. Using this interpretation we find a set of analytical expressions for conditions that the Luttinger parameters must satisfy to avoid spurious solutions. Finally, we use these conditions to check commonly used sets of parameters for their potential for generating this class of spurious solutions. (C) 2003 American Institute of Physics. [DOI: 10.1063/1.1555833]
\end{abstract}

\section{INTRODUCTION}

The effective mass approximation (EMA) ${ }^{1,2}$ has long been a favorite among researchers as a fast and easy to implement method for obtaining the energy levels in quantum wells and superlattices, ${ }^{3-7}$ quantum wires, ${ }^{8-10}$ and quantum dots, ${ }^{8,11,12}$ etc. It is also the tool of choice for engineers to model devices such as lasers and photodetectors. ${ }^{13-18}$ Also, its use is widespread in the calculation of transmission coefficients ${ }^{19-21}$ and quasibound state lifetimes, ${ }^{22,23}$ with application to the modeling of resonant tunneling devices.

Some of the implementations have the undesirable characteristic that they produce spurious solutions; ${ }^{3,24-26}$ their origin has been traced to the statement of a secular equation having too high a polynomial degree in the electron wave vector $k$. In some cases, the presence of the spurious solutions is required for consistent boundary conditions at the interface to be satisfied, ${ }^{3}$ thus raising doubts about the validity of the results. ${ }^{24}$ In some other cases, interface or surface states are predicted to lie in the gap, ${ }^{27}$ but the physical meaning of these solutions is still a subject of discussion. ${ }^{28}$ There exist several proposals to solve the $\mathbf{k} \cdot \mathbf{p}$ spurious solution problem ranging from methods to eliminate them ${ }^{29,30}$ to pointing out the necessity of keeping them for a complete description. ${ }^{31}$

The finite difference method (FDM) has been used extensively to solve the EMA equations ${ }^{32-36}$ because external fields can be included straightforwardly in this formalism. In this article a class of spurious solutions (SSs) particular to the FDM is studied. In Sec. II the problem under study is stated, and the behavior of the SSs is characterized under changing conditions. Then, in Sec. III A, a general method for the study of this class of SSs is derived. In Sec. III B a set

a) Author to whom correspondence should be addressed; electronic mail: tcm@ssdp.caltech.edu of analytical expressions that the Luttinger parameters must satisfy to avoid the SSs is obtained. Finally, in Sec. IV B this condition is applied to popular compilations of Luttinger parameters to identify sets of parameters leading to SSs.

\section{CHARACTERIZATION OF THE SPURIOUS SOLUTIONS}

We illustrate the spurious solutions (SSs) in an $\mathrm{InP} / \mathrm{In}_{0.53} \mathrm{Ga}_{0.47}$ As symmetric superlattice (SL) grown along the [001] direction with an $\operatorname{In}_{0.53} \mathrm{Ga}_{0.47} \mathrm{As}$ width of $65 \AA$ and an InP width of $88 \AA$. The method of calculation is the standard eight-band EMA with Chuang and Chang's discretization scheme. ${ }^{34}$ Table I lists the numerical parameters employed in the calculations. When there is no explicit source for the parameters of $\operatorname{In}_{0.53} \mathrm{Ga}_{0.47} \mathrm{As}$, they have been obtained as a linear interpolation between InAs and GaAs, except for the eight-band Luttinger parameters $\gamma_{i}$, where a harmonic average has been performed. ${ }^{37}$ The true Luttinger parameters - denoted by a subscript $L$ - and the modified (aka. eight-band, Kane) Luttinger parameters are related by ${ }^{38}$

$$
\begin{aligned}
& \gamma_{1}=\gamma_{1 L}-\frac{1}{3} \frac{E_{P}}{E_{g}}, \\
& \gamma_{2}=\gamma_{2 L}-\frac{1}{6} \frac{E_{P}}{E_{g}}, \\
& \gamma_{3}=\gamma_{3 L}-\frac{1}{6} \frac{E_{P}}{E_{g}},
\end{aligned}
$$

where $E_{g}$ is the energy gap of the compound, and $E_{P}$ has been defined as $E_{P} \equiv 2 m P^{2} / \hbar^{2}$, with $m$ being the free electron mass and $P$ the interband momentum matrix element.

Figure 1(a) shows the band edge spatial profile for that structure. Figure 1(b) shows a calculation of a few subbands near the conduction band edge of the SL. The energy scales 
TABLE I. Band structure parameters for $\mathrm{InP}$ and $\operatorname{In}_{0.53} \mathrm{Ga}_{0.47} \mathrm{As}$.

\begin{tabular}{ccccccccc}
\hline \hline & $a^{\mathrm{c}}$ & $E_{g}(\mathrm{eV})$ & $E_{v}(\mathrm{eV})$ & $\Delta_{\mathrm{SO}}(\mathrm{eV})$ & $\gamma_{1}$ & $\gamma_{2}$ & $\gamma_{3}$ & $P(\mathrm{eV} \AA)$ \\
\hline $\mathrm{InP}^{\mathrm{a}}$ & 5.8693 & 1.35 & -0.351 & 0.108 & 1.49 & -0.31 & 0.37 & 8.79 \\
$\mathrm{In}_{0.53} \mathrm{Ga}_{0.47} \mathrm{As}^{\mathrm{b}}$ & 5.8693 & 0.839 & 0 & 0.362 & $1.84^{\mathrm{d}}$ & $-0.87^{\mathrm{d}}$ & $0.25^{\mathrm{d}}$ & 9.49 \\
\hline \hline
\end{tabular}

${ }^{\mathrm{a}}$ Reference 45 unless noted.

${ }^{\mathrm{b}}$ Obtained by linear interpolation unless noted.

'Reference 47.

${ }^{\mathrm{d}}$ Obtained by harmonic averaging (Ref. 37).

in plots (a) and (b) are matched. Note the presence of spurious solutions, with the energies lying inside the gap of the $\operatorname{In}_{0.53} \mathrm{Ga}_{0.47} \mathrm{As}$.

The relationship of the SSs to the discretization grid is investigated in Fig. 2. There, the band edge energies are plotted versus the number of mesh points. Four SSs appear in the range of mesh points and energies under study. It is seen that while, as expected, the energies of the physical states do not depend strongly on the number of mesh points, ${ }^{39}$ the energies of the SSs do depend on the number of mesh points. After this, it must be concluded that the SSs presented here are not only nonphysical, but also that they are not intrinsic properties of the system of coupled differential equations

$$
\mathbf{H}\left(\mathbf{k}_{\|},-i \partial_{z}\right) \mathbf{F}(z)=E \mathbf{F}(z)
$$

-where $\mathbf{H}$ is the Hamiltonian, $E$ is the energy, and $\mathbf{F}(z)$ is the $z$-dependent envelope function-that must be solved in the EMA model. ${ }^{1}$ Instead, they appear only due to the discretization procedure followed to solve these equations.

One can obtain more insight into the nature of these spurious solutions by plotting the norm and the relevant en- velope function components of the wave function corresponding to the highest lying SS. This is done in Fig. 3(a) along with a comparison to a physical wave function [plot (b)]. It can be seen that the probability density $F(z)$ $=\sum_{i=1}^{8}\left|F_{i}(z)\right|^{2}$ of finding the electron in a given monolayer is similar in both cases and does not supply any information. However, a look at the individual components of the envelope function reveals that they are highly oscillatory for the spurious case, while they are smooth for the physical state. The fact that the states are located mainly in the center of the $\mathrm{In}_{0.53} \mathrm{Ga}_{0.47}$ As layer discards the possibility that the SSs might originate from boundary condition induced interface states, ${ }^{28,40}$ because the latter should decay exponentially away from the interface.

Also, the fact that the envelope functions are oscillating in the whole $\mathrm{In}_{0.53} \mathrm{Ga}_{0.47} \mathrm{As}$ region indicates that the spurious solutions are a consequence of the method even in bulk materials rather than being somehow connected to the boundary conditions. The oscillation period associated with the wave functions of the SSs is always twice the mesh spacing, inde- a)

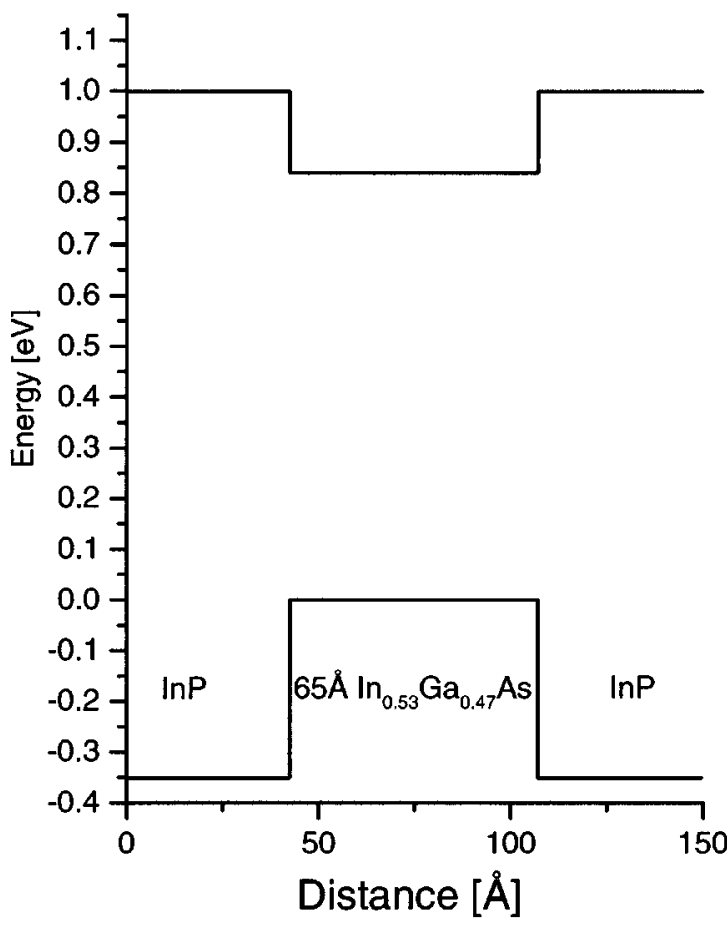

b)

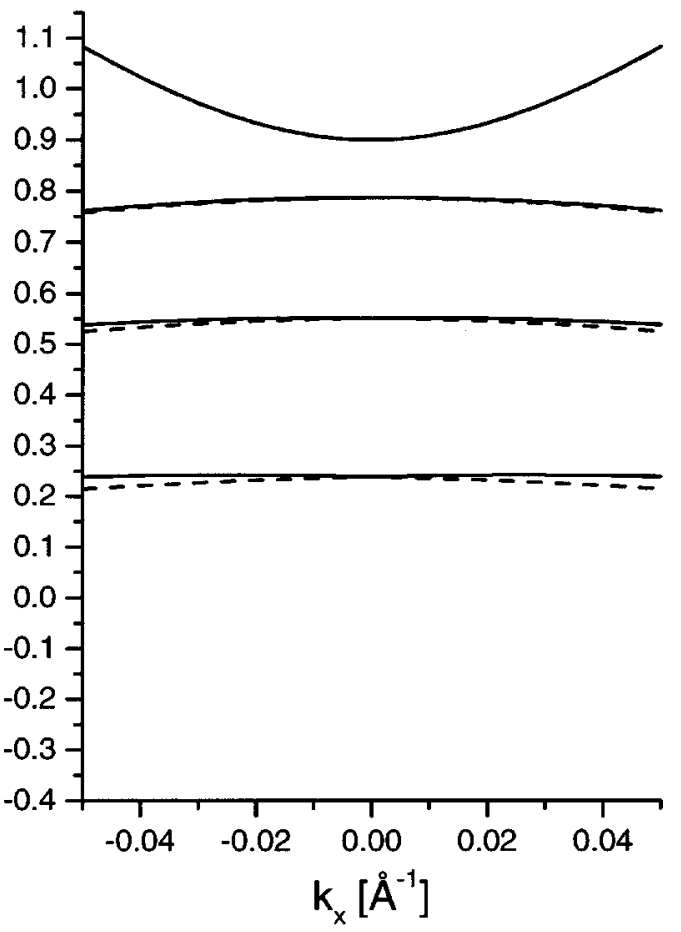

FIG. 1. Bands of an $88 \AA / 65 \AA$ InP/In ${ }_{0.53} \mathrm{Ga}_{0.47} \mathrm{As}$ SL showing spurious solutions. (a) The spatial profile of the bulk bands. (b) The bands in $\mathbf{k}$ space along [100] in the same energy scale as in (a). It is seen that the first three hole bands lie in the forbidden gap. 


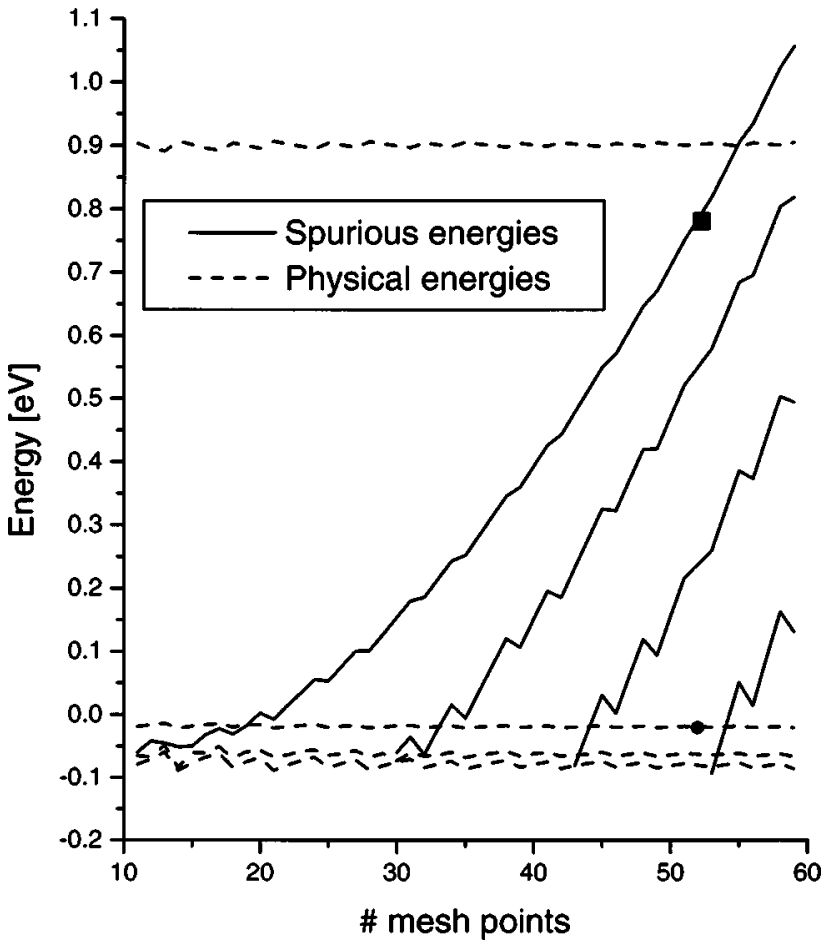

FIG. 2. Energy of the spurious states vs number of mesh points for an 88 $\AA / 65 \AA \mathrm{InP} / \mathrm{In}_{0.53} \mathrm{Ga}_{0.47} \mathrm{As} \mathrm{SL}$. The state labeled with the square (round) dot corresponds to plot (a) [(b)] in Fig. 3.

pendent of the mesh size, and is highly reminiscent of the properties of $X$-valley derived states in tight-binding calculations. This leads us to suspect that the bulk band structure of $\mathrm{In}_{0.53} \mathrm{Ga}_{0.47} \mathrm{As}$, as computed in the finite difference method, may contain nonphysical satellite valleys, which could lead to spurious solutions. As will be demonstrated later, calculations with bulk $\mathrm{In}_{0.53} \mathrm{Ga}_{0.47}$ As indeed show this behavior.

\section{METHODS}

In solving the EMA equations using the FDM, we implicitly modify the band structure from one given by the EMA equations directly to the finite difference band structure. While the band structures computed from the two different methods are almost identical near the zone center, where wavelengths are large compared to mesh spacing, substantial deviations can occur at larger $k$ vectors. There is no $a$ priori guarantee that the FDM band structure would be well behaved away from the zone center and an ill-behaved FDM bulk band structure can lead to spurious solutions. In this section we will describe the methods we use to analyze SSs in FDM.

\section{A. General method for studying spurious solutions in discretized bases}

Several practical implementations of calculations of band structures of heterostructures using spatially discretized basis sets, including the multiband $\mathbf{k} \cdot \mathbf{p}^{33,34}$ and effective bond-orbital model ${ }^{41}$ methods, require the solution of a set of equations

$$
\mathbf{H}_{\sigma, \sigma-1} \mathbf{F}_{\sigma-1}+\mathbf{H}_{\sigma, \sigma} \mathbf{F}_{\sigma}+\mathbf{H}_{\sigma, \sigma+1} \mathbf{F}_{\sigma+1}=E \mathbf{F}_{\sigma}
$$

for each of the points $\{\sigma\}$ in the mesh, where $\mathbf{F}_{\sigma}$ are the envelope function coefficients at the mesh point $\sigma$ and the $\mathbf{H}_{\sigma, \nu}$ are matrices containing on-site and hopping elements of the Hamiltonian.

In bulk, the envelope functions of the physical solutions at the $\Gamma$ point should be flat. On the other hand, it has been shown in the previous section that SSs oscillate with a period of double the mesh spacing. Both cases can be studied if one assumes that the envelope must follow the Bloch behavior, relating the value of the envelope at a point $\sigma+1$ to the value at $\sigma$

$$
\mathbf{F}_{\sigma+1}=e^{i \Delta z k_{z}} \mathbf{F}_{\sigma},
$$

where $\Delta z$ is the mesh spacing and $k_{z}$ is the $z$ component of the electron wave vector in bulk.

Now, plugging Eq. (4) into Eq. (3), one obtains a single eigenvalue equation

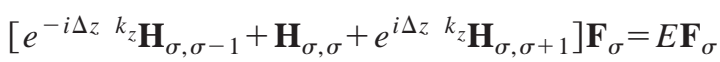

that must be solved in order to know the effect of an oscillating envelope function on the energies. Any further advance requires an explicit form for the Hamiltonian.

\section{B. Spurious solutions in the $k \cdot p$ method}

In the eight-band EMA method, the starting point is a model that treats the coupling between the conduction band (CB), heavy hole (HH), light hole (LH), and split-off (SO) bands exactly, and the interactions with the rest of the bands perturbatively. Bulk inversion asymmetry effects ${ }^{42}$ will be ignored to keep the results simple and obtain analytical expressions. This will cause the bands to be doubly degenerate. The diagonalization of Eq. (5) can be carried out numerically if some of the assumptions need to be relaxed. Also, since only spurious solutions at the heterostructure zone center are sought, the $k_{x}$ and $k_{y}$ components of the wave vector are set to zero. Then the k·p Hamiltonian diagonalizes into two 4 $\times 4$ blocks, and each one of them is block diagonalized again into a $1 \times 1$ block describing the $\mathrm{HH}$ band and a $3 \times 3$ block describing the $\mathrm{CB}, \mathrm{LH}$ and $\mathrm{SO}$ bands

$H_{3 \times 3}$

$$
{ }_{\mathrm{CB}}\left\langle+|+| \begin{array}{ccc}
|+\rangle_{\mathrm{CB}} & |+\rangle_{\mathrm{LH}} & |+\rangle_{\mathrm{SO}} \\
E_{g}+E_{z} & \sqrt{\frac{2}{3}} P k_{z} & -\frac{P k_{z}}{\sqrt{3}} \\
\sqrt{\frac{2}{3} P k_{z}} & -\left(\gamma_{1}+2 \gamma_{2}\right) E_{z} & \sqrt{2} \gamma_{2} E_{z} \\
-\frac{P k_{z}}{\sqrt{3}} & \sqrt{2} \gamma_{2} E_{z} & -\Delta_{\mathrm{SO}}-\gamma_{1} E_{z}
\end{array}\right),
$$

where $E_{z} \equiv \hbar^{2} k_{z}^{2} / 2 m$ and $\Delta_{\text {SO }}$ is the split-off splitting.

After the application of the discretization procedure described in Ref. 34 to Eq. (6) and plugging the result into Eq. (5) one obtains that the finite difference algorithm is effectively solving the Hamiltonian 
a)
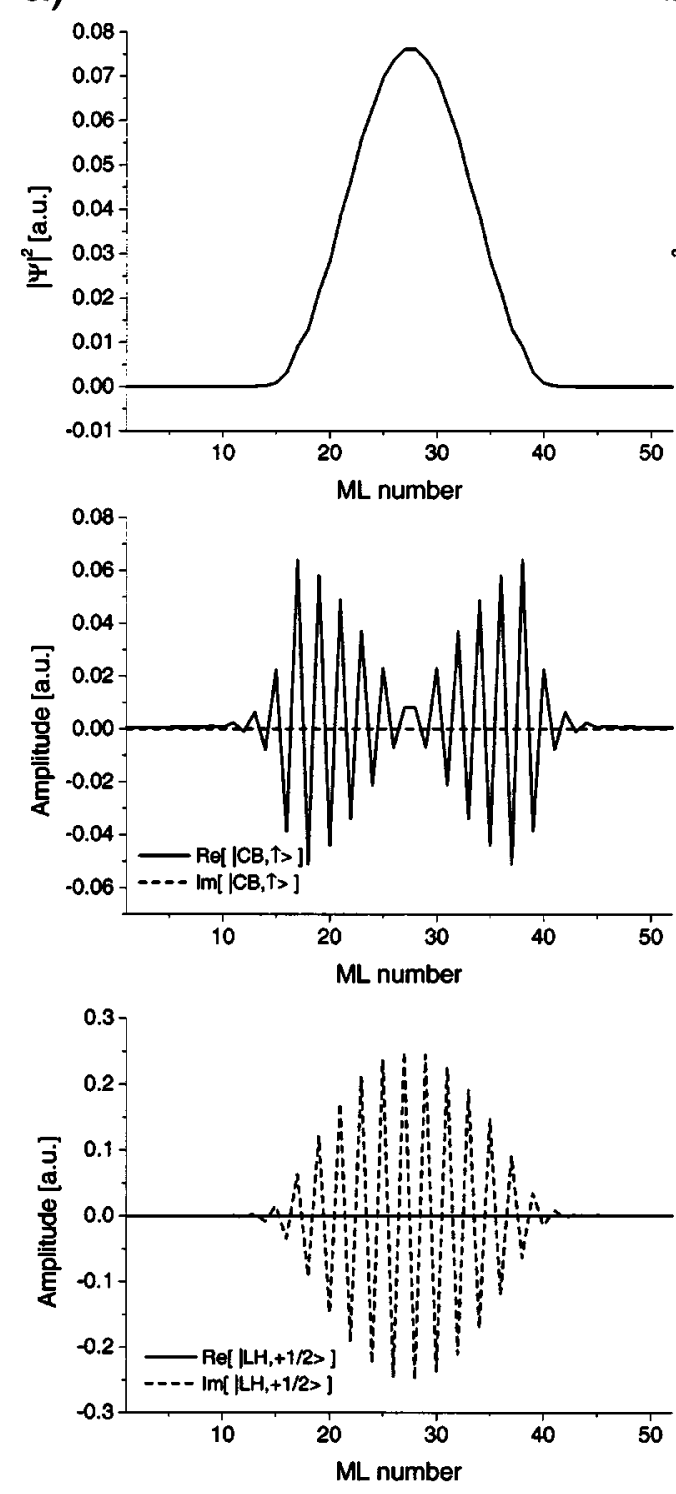

b)
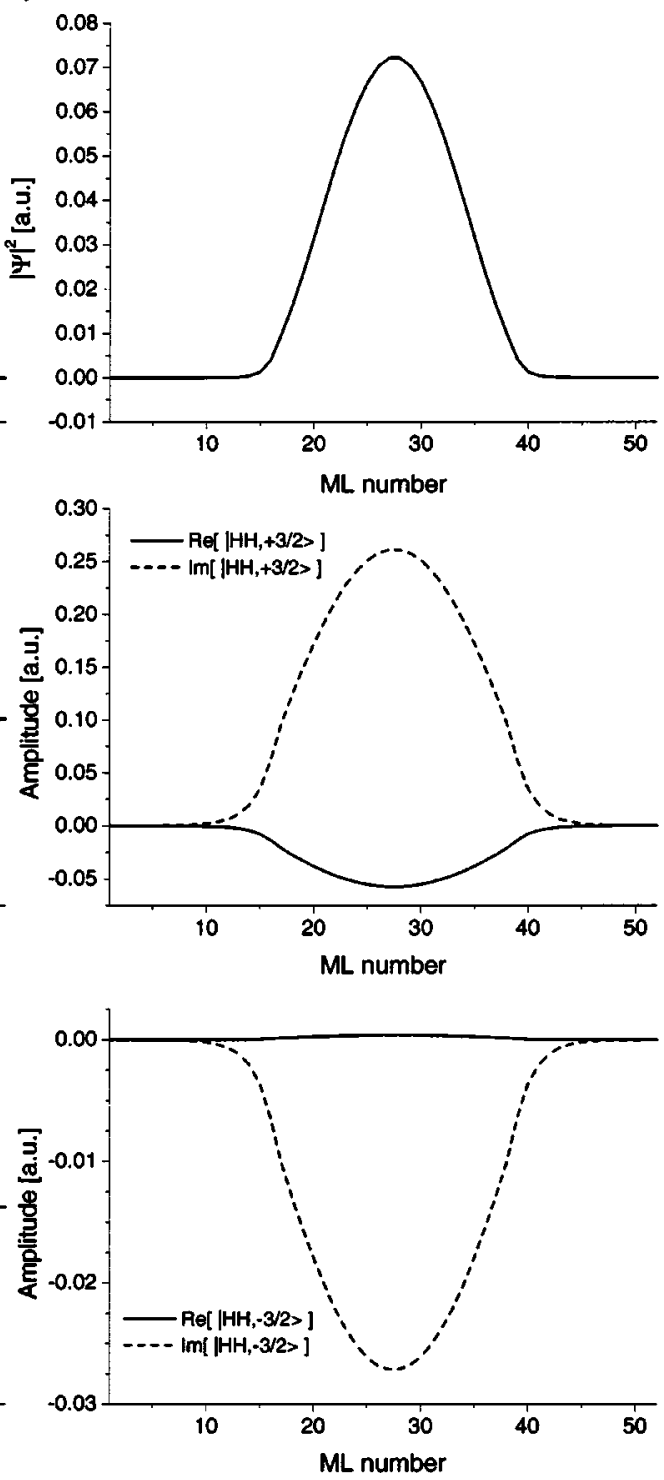

FIG. 3. Comparison of spurious and physical envelope functions. (a) Corresponds to a spurious state. (b) Corresponds to the first heavy hole state. The top plots correspond to the probability density of finding the electron in a given monolayer, while the bottom two show the real and imaginary parts of selected envelope components.

$$
H_{3 \times 3 \mathrm{FDM}}=\left(\begin{array}{ccc}
E_{g}+\frac{\hbar^{2}}{m} \frac{1-\cos \left(\Delta z k_{z}\right)}{\Delta z^{2}} & \frac{\sqrt{\frac{2}{3}} P \sin \left(\Delta z k_{z}\right)}{\Delta z} & -\frac{P \sin \left(\Delta z k_{z}\right)}{\sqrt{3} \Delta z} \\
\frac{\sqrt{\frac{2}{3}} P \sin \left(\Delta z k_{z}\right)}{\Delta z} & -\left(\gamma_{1}+2 \gamma_{2}\right) \frac{\hbar^{2}}{m} \frac{1-\cos \left(\Delta z k_{z}\right)}{\Delta z^{2}} & \frac{\hbar^{2}}{m} \frac{2 \sqrt{2} \gamma_{2} \sin \left(\frac{\Delta z k_{z}}{2}\right)^{2}}{\Delta z^{2}} \\
-\frac{P \sin \left(\Delta z k_{z}\right)}{\sqrt{3} \Delta z} & \frac{\hbar^{2}}{m} \frac{2 \sqrt{2} \gamma_{2} \sin \left(\frac{\Delta z k_{z}}{2}\right)^{2}}{\Delta z^{2}} & -\Delta_{\mathrm{SO}}-\gamma_{1} \frac{\hbar^{2}}{m} \frac{1-\cos \left(\Delta z k_{z}\right)}{\Delta z^{2}}
\end{array}\right) .
$$


a)

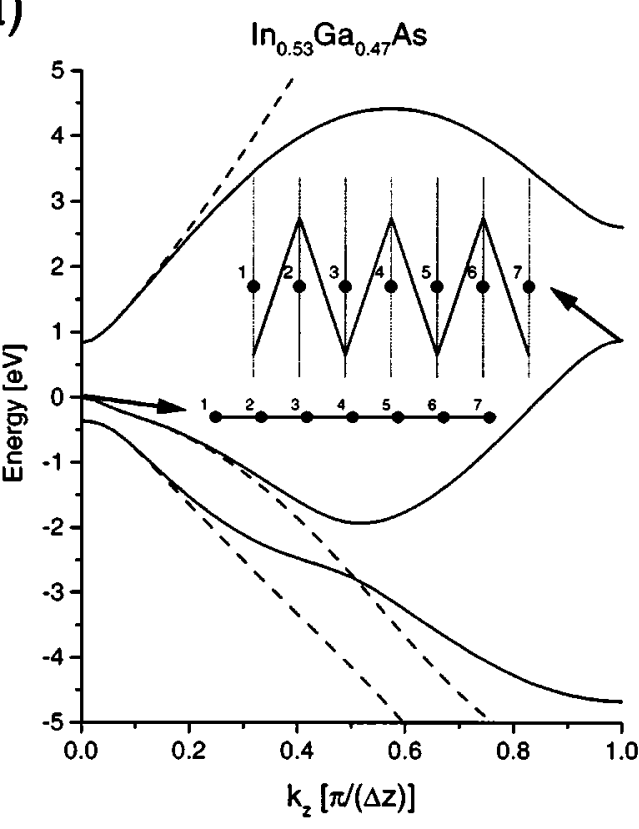

b)

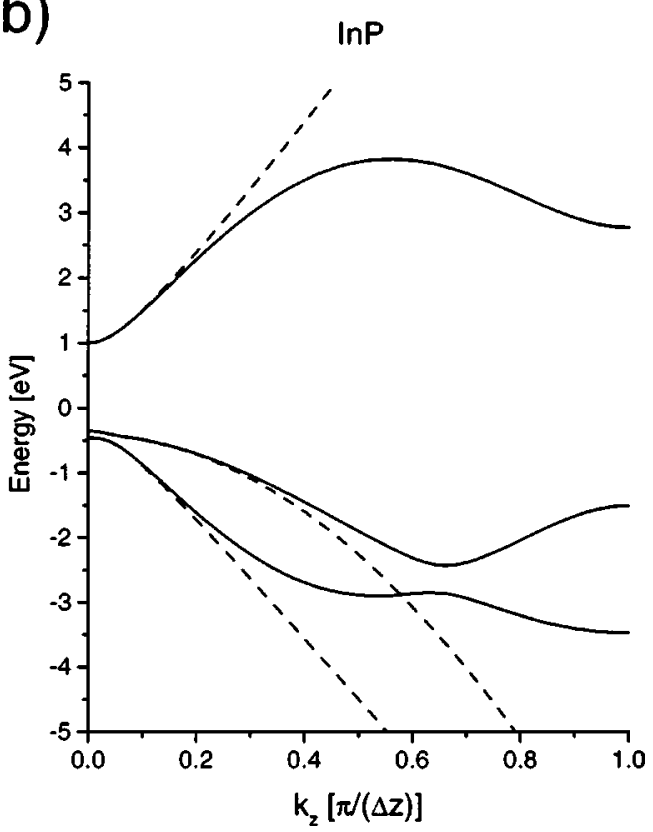

FIG. 4. Energy vs wave number of a hypothetical spurious solution. (a) [(b)] shows the dispersion relation for $\operatorname{In}_{0.53} \mathrm{Ga}_{0.47} \mathrm{As}$ (InP) with $\Delta z$ equal to half the unit cell size. The insets show two kinds of light hole envelope functions $\left(k_{z}=0\right.$ and $\left.k_{z}=\pi / \Delta z\right)$ satisfying cyclic boundary conditions. The solid lines are calculated with Eq. (7) (FDM band structure), while the dashed lines are obtained with Eq. (6) (k·p band structure).

Expanding this Hamiltonian about $k_{z}=0$ up to second order it is easily seen that Eq. (6) is recovered, which ensures the correct description of the bands when $\Delta z k_{z} \ll 1$.

Now, the eigenvalues of Eq. (7) can be plotted as a function of $k_{z}$ for both the InP and the $\mathrm{In}_{0.53} \mathrm{Ga}_{0.47}$ As parameters in Table I. A comparison of the EMA and FDM band structures of $\mathrm{In}_{0.53} \mathrm{Ga}_{0.47} \mathrm{As}$ in Fig. 4(a) immediately reveals the origin of the spurious solutions. The FDM band structure for $\mathrm{In}_{0.53} \mathrm{Ga}_{0.47} \mathrm{As}$ contains a local maximum at the zone boundary or $X$ point (as defined with respect to the specific mesh spacing used). When the quantized states of this inverted $X$ valley are zone folded, they appear as the SSs observed. This is why the spurious solutions exhibit the oscillations that are characteristic of $X$-point derived states. The insets of Fig. 4(a) show the $\Gamma$ valley- and the $X$-valley-derived wave functions over six periods of bulk material. The existence of the $X$-valley-derived solutions is unavoidable in the FDM but, at least, one can demand that they lie far from the energy range of interest. This is indeed the case with InP in Fig. 4(b) but, on the other hand, the LH band of $\operatorname{In}_{0.53} \mathrm{Ga}_{0.47}$ As enters the gap, thus giving opportunity to the presence of SSs in the midgap.

Also, one finds that, for $k_{z}=\pi / \Delta z$, the energy of the three branches (with $E_{v}$ set to zero) is

$$
\begin{aligned}
\left\{E_{g}\right. & +\frac{2 \hbar^{2}}{m \Delta z},-\frac{4\left(\gamma_{1}+\gamma_{2}\right) \hbar^{2} / m+\Delta_{\mathrm{SO}}(\Delta z)^{2}}{2(\Delta z)^{2}} \\
& \left. \pm \frac{\sqrt{48 \gamma_{2}^{2} \hbar^{4} / m^{2}-8(\Delta z)^{2} \gamma_{2} \Delta_{\mathrm{SO}} \hbar^{2} / m+\Delta_{\mathrm{SO}}^{2}(\Delta z)^{4}}}{2(\Delta z)^{2}}\right\} .
\end{aligned}
$$

One can argue that, since the well region in the SL is quite wide, the first electron and hole levels will be close to the corresponding edges. Then, the analytic expression corre- sponding to the plus sign in front of the square root in Eq. (8), describing the energy of the $k_{z}=\pi / \Delta z$ light hole (LH) states in bulk $\mathrm{In}_{0.53} \mathrm{Ga}_{0.47}$ As can be used to make a rough approximation of the energy of the SS in the superlattice (SL) studied in Fig. 2. Figure 5 shows a comparison of the energy of the SS estimated this way and the highest energy SS from the calculation in Fig. 2. The agreement is quite good, and it supports the claim that the mechanism presented in the previous and the present sections is responsible for the appearance of this class of mesh-dependent SSs. It is expected that a similar study to the one in Sec. III A, but extended this way

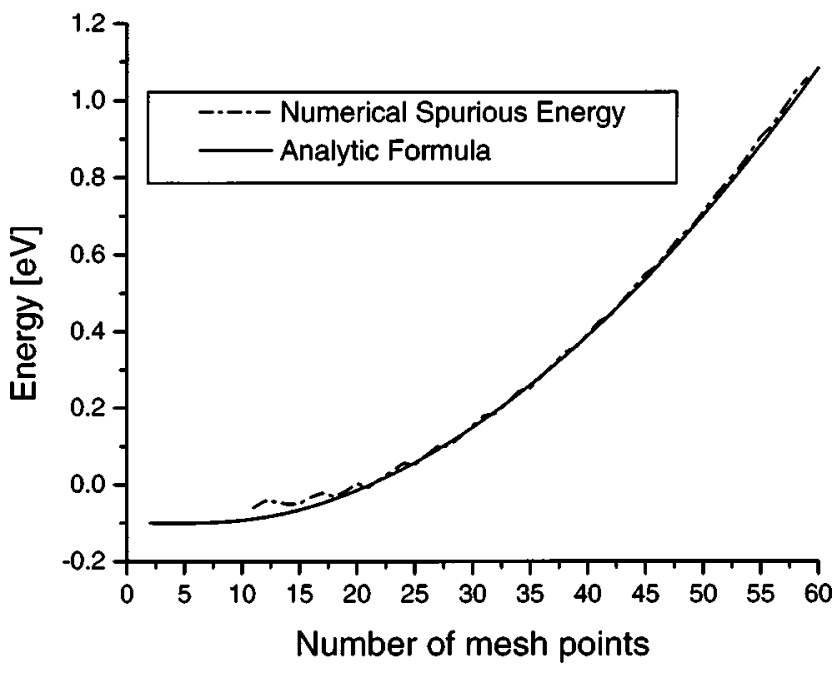

FIG. 5. Prediction from Eq. (8) and actual spurious solution energy. The actual spurious energy corresponds to the highest spurious energy in Fig. 2. 


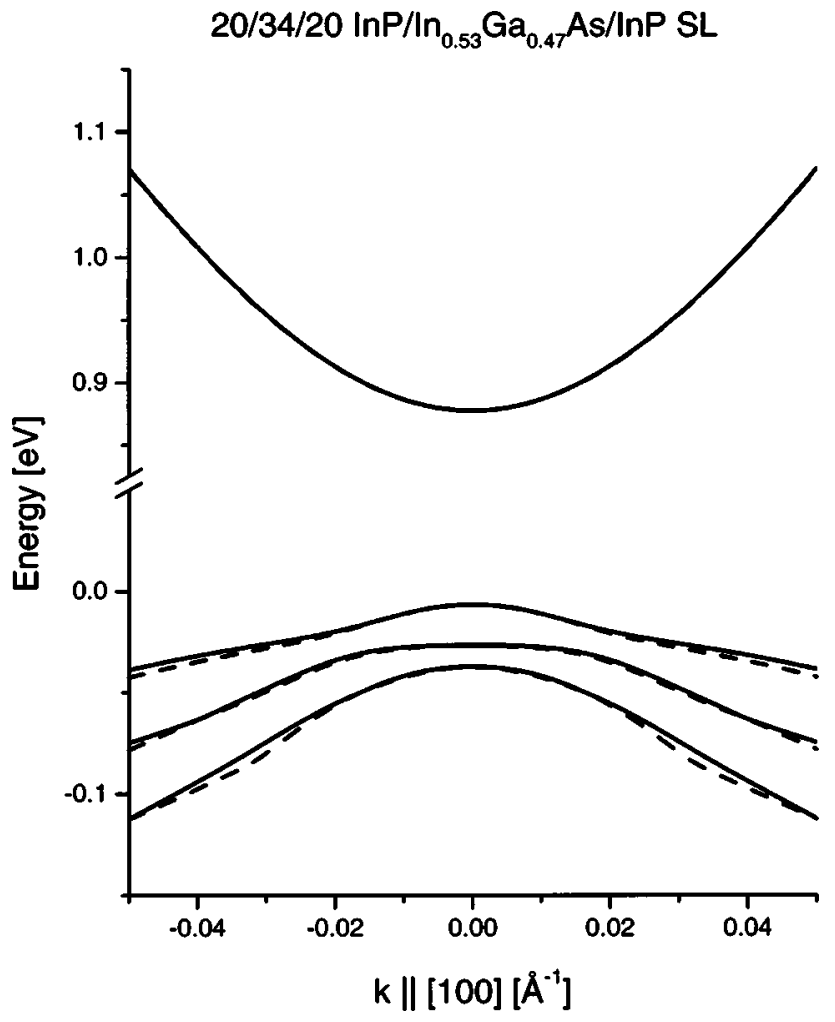

FIG. 6. Bands of an $88 \AA / 65 \AA \operatorname{InP} / \mathrm{In}_{0.53} \mathrm{Ga}_{0.47} \mathrm{As}$ SL showing no spurious solutions.

$$
\begin{aligned}
& \mathbf{H}_{\sigma, \sigma-1} \mathbf{F}_{\sigma-1}+\mathbf{H}_{\sigma, \sigma} \mathbf{F}_{\sigma}+\mathbf{H}_{\sigma, \sigma+1} \mathbf{F}_{\sigma+1}=E \mathbf{F}_{\sigma}, \\
& \mathbf{H}_{\sigma+1, \sigma} \mathbf{F}_{\sigma}+\mathbf{H}_{\sigma+1, \sigma+1} \mathbf{F}_{\sigma+1}+\mathbf{H}_{\sigma+1, \sigma+2} \mathbf{F}_{\sigma+2}=E \mathbf{F}_{\sigma+1}
\end{aligned}
$$

and keeping Eq. (4) could describe lower energy SSs. The condition that we derive below is expected at least to delay the appearance of these lower energy SSs.

The SSs will first originate from the eigenvalue in Eq. (8) with the plus sign in front of the square root (i.e., the LH band). A reasonable requirement to avoid solutions in the gap is that the LH energy for $k_{z}=\pi / \Delta z$ be less than the valence band edge (which is set to zero). Therefore, one wants

$$
\begin{aligned}
& \sqrt{48 \gamma_{2}^{2} \hbar^{4} / m^{2}-8(\Delta z)^{2} \gamma_{2} \Delta_{\mathrm{SO}} \hbar^{2} / m+\Delta_{\text {SO }}^{2}(\Delta z)^{4}} \\
& \quad<4\left(\gamma_{1}+\gamma_{2}\right) \hbar^{2} / m+\Delta_{\text {SO }}(\Delta z)^{2} .
\end{aligned}
$$

Following the algebra on that expression and assuming $\gamma_{1}+2 \gamma_{2}>0$, one gets to the condition that the mesh spacing must satisfy in order to ensure that there are no solutions in the gap is obtained

$$
(\Delta z)^{2}>\frac{2\left(2 \gamma_{2}^{2}-2 \gamma_{1} \gamma_{2}-\gamma_{1}^{2}\right) \frac{\hbar^{2}}{m}}{\Delta_{\mathrm{SO}}\left(\gamma_{1}+2 \gamma_{2}\right)} .
$$

This condition will always be satisfied if the right hand side of that inequality is less than zero. So this yields the condition that the modified Luttinger parameters must satisfy in order to avoid SSs in the FDM method for any mesh spacing
TABLE II. Luttinger parameters for $\operatorname{In}_{0.53} \mathrm{Ga}_{0.47}$ As satisfying the condition for the absence of SSs (adapted from Ref. 43).

\begin{tabular}{cccc}
\hline \hline & $\gamma_{1}$ & $\gamma_{2}$ & $\gamma_{3}$ \\
\hline $\mathrm{In}_{0.53} \mathrm{Ga}_{0.47} \mathrm{As}$ & 1.63 & -0.27 & -1.37 \\
\hline & & & \\
$\frac{1-\sqrt{3}}{2} \gamma_{1}<\gamma_{2}<\frac{1+\sqrt{3}}{2} \gamma_{1}$. & & (13)
\end{tabular}

If the above condition is not satisfied for a material, Eq. (12) can be used to find a safe mesh spacing. Of course, for some choice of parameters the safe mesh spacing will be too big for the solutions to be accurate. In that case, a different set of parameters or a different solution method for the EMA equations should be employed.

Now, in the case that $\gamma_{1}+2 \gamma_{2}<0$, it is easy to see that $\Delta z$ must satisfy

$$
(\Delta z)^{2}<\frac{2\left(2 \gamma_{2}^{2}-2 \gamma_{1} \gamma_{2}-\gamma_{1}^{2}\right) \frac{\hbar^{2}}{m}}{\Delta_{\mathrm{SO}}\left(\gamma_{1}+2 \gamma_{2}\right)},
$$

which can only be satisfied if the right hand side is positive, leading to the conditions (13) again.

Finally, imposing that the branch with the minus sign in front of the square root in Eq. (8) (the spin-orbit band) also has a negative energy at $k_{z}=\pi / \Delta z$ will result in the same set of conditions (13).

\section{RESULTS}

\section{A. The $\mathrm{InP} / \mathrm{In}_{0.53} \mathrm{Ga}_{0.47}$ As SL revisited}

Looking back at the parameters for $\mathrm{In}_{0.53} \mathrm{Ga}_{0.47} \mathrm{As}$ in Table I, it is seen that $(1-\sqrt{3}) / 2 \gamma_{1}=-0.67>-0.87=\gamma_{2}$. Therefore, the conditions for the absence of spurious solutions in the gap were not satisfied. For that case, any mesh spacing smaller than $22.4 \AA$ would have triggered the appearance of a spurious solution close to or in the gap.

A different set of Luttinger parameters for $\mathrm{In}_{0.53} \mathrm{Ga}_{0.47} \mathrm{As}$ was found ${ }^{43}$ and the band structure of the $\mathrm{InP} / \mathrm{In}_{0.53} \mathrm{Ga}_{0.47} \mathrm{As}$

\begin{tabular}{|c|c|c|c|c|c|}
\hline & $\gamma_{1}$ & $\gamma_{2}$ & $\gamma_{3}$ & $\frac{1-\sqrt{3}}{2} \gamma_{1}$ & $\frac{1+\sqrt{3}}{2} \gamma$ \\
\hline AlP & 2.32 & -0.52 & 0.57 & -0.85 & 3.17 \\
\hline AlAs & 1.74 & -0.37 & 0.42 & -0.64 & 2.38 \\
\hline $\mathrm{AlSb}$ & 1.44 & -0.35 & 0.39 & -0.53 & 1.97 \\
\hline $\mathrm{GaP}$ & 1.62 & -0.31 & 0.37 & -0.59 & 2.22 \\
\hline GaAs & 2.01 & -0.41 & 0.46 & -0.74 & 2.75 \\
\hline $\mathrm{GaSb}$ & 2.58 & -0.58 & 0.65 & -0.95 & 3.53 \\
\hline $\operatorname{InP}$ & 1.49 & -0.31 & 0.37 & -0.55 & 2.04 \\
\hline InAs & 2.05 & -0.44 & 0.48 & -0.75 & 2.80 \\
\hline $\mathrm{InSb}$ & 2.59 & -0.60 & 0.67 & -0.95 & 3.54 \\
\hline
\end{tabular}
superlattice recalculated. These bands are plotted in Fig. 6, and they show no spurious solutions. The modified Luttinger parameters used are listed in Table II.

TABLE III. Check for possibility of spurious solutions in the Luttinger parameters from Lawaetz (see Ref. 45). 
TABLE IV. Check for possibility of spurious solutions in the Luttinger parameters from Vurgaftman, Meyer, and Ram-Mohan (see Ref. 44).

\begin{tabular}{lcccccc}
\hline \hline & & & & $\frac{1-\sqrt{3}}{2} \gamma_{1}$ & $\frac{1+\sqrt{3}}{2} \gamma_{1}$ & Ill conditioned \\
\hline AlP & 1.72 & -0.10 & 0.42 & -0.63 & 2.36 & No \\
AlAs & 1.49 & -0.31 & 0.29 & -0.55 & 2.04 & No \\
AlSb & 2.57 & -0.12 & 0.66 & -0.94 & 3.51 & No \\
$\mathrm{GaP}$ & 0.42 & -1.32 & 1.12 & -0.15 & 0.58 & Yes \\
$\mathrm{GaAs}$ & 0.66 & -1.10 & -0.23 & -0.24 & 0.90 & Yes \\
$\mathrm{GaSb}$ & 2.32 & -0.84 & 0.46 & -0.85 & 3.16 & No \\
$\mathrm{InP}$ & 0.23 & -0.82 & -0.32 & -0.09 & 0.32 & Yes \\
$\mathrm{InAs}$ & 2.81 & -0.09 & 0.61 & -1.03 & 3.84 & No \\
InSb & 1.75 & -1.02 & -0.02 & -0.64 & 2.39 & Yes \\
\hline
\end{tabular}

${ }^{\mathrm{a}}$ As described in the text and in Fig. 7(b), not advisable to use.

\section{B. Potential for spurious solution in Luttinger parameter sets}

A great amount of literature can be found about calculations and measurement of Luttinger parameters for compounds and alloys. A comprehensive review was published by Vurgaftman and co-workers. ${ }^{44}$ Another commonly used list of parameters was tabulated by Lawaetz. ${ }^{45}$ In this section the condition in Eq. (13) will be applied to the listed parameters in the above two references for selected materials to detect the potential presence of SSs when using those data.

Table III lists the eight-band Luttinger parameters adapted from Lawaetz ${ }^{45}$ for a set of III-Vs and the limits of the interval where $\gamma_{2}$ must lie in order to avoid SSs. It is seen that none of those sets of parameters present a potential for SSs.

However, the situation for alloys requires a more careful consideration. A possible approach to obtain the modified Luttinger parameters for alloys such as $\operatorname{In}_{x} \mathrm{Ga}_{1-x} \mathrm{As}$ or
$\mathrm{Al}_{x} \mathrm{Ga}_{1-x} \mathrm{As}$ is to take averages of the parameters of the base compounds. If this is the approach followed, the calculated parameters are not at risk of producing SSs. On the other hand, another plausible approach would be to take the direct $^{46}$ or reciprocal ${ }^{37}$ averages of the true Luttinger parameters instead, and then use Eqs. (1) to find the modified parameters. Since the k.p Hamiltonian is mostly expressed in terms of the true Luttinger parameters, the averages of these are mainly used in the literature. Nevertheless, a linear interpolation for an alloy of all the terms in Eq. (1) can introduce considerable bowing in the modified parameters, so one should be careful when calculating modified parameters using the latter approach and make sure to check that the condition (13) is satisfied. An experimental study of the hole effective masses as a function, say, of the Ga composition, and then finding from there the modified parameters should be able to discern which one of the two approaches is more accurate or whether a more complex interpolation formula should be used.

The bulk Luttinger parameters tabulated for III-Vs in Vurgaftman and co-workers ${ }^{44}$ are more dangerous to use in a FDM implementation of the EMA. Table IV shows the modified parameters calculated from the true Luttinger parameters in Ref. 44. It is easy to check that GaP, GaAs, InP and InSb do not satisfy Eq. (13), and that GaSb is close to the lower limit. Figure 7 shows the energy of the states given by Eq. (4) for GaAs and GaSb computed with Eq. (7) using parameters from Ref. 44. Again, the light hole and split-off bands start bending downward reproducing the physical effective mass, but at about $k_{z}=\pi / 2 \Delta z$ the LH band bends up and enters the gap region for GaAs and finishes close to it for $\mathrm{GaSb}$. In the case of GaAs there is evident risk that these solutions could fold into midgap SSs in a GaAs based het-
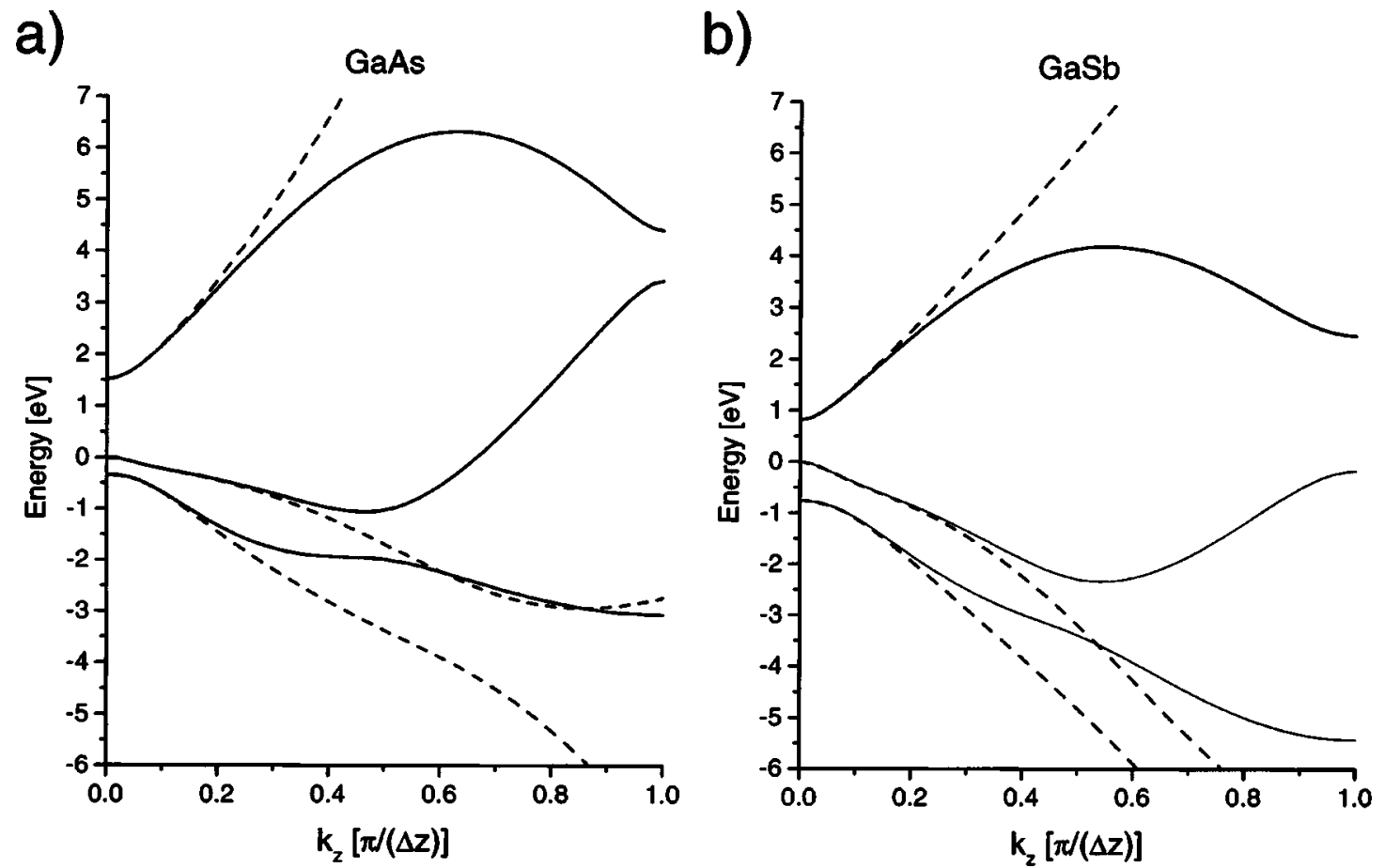

FIG. 7. Plot (a) [(b)] is the same as Fig. 4 but for GaAs $(\mathrm{GaSb})$ with Luttinger parameters from Ref. 44 . 
erostructure. The set of GaSb parameters is not ideal for the FDM, either, because the energy of the LH solution at $k_{z}$ $=\pi / \Delta z$ is too close to the valence band edge [see Fig. 7(b)]. This state might interfere with states mainly in the GaSb layer in a quantum well or superlattice. We stress that these spurious solutions are particular to the FDM. As can be seen in Fig. 7, the Vurgaftman parameters are perfectly safe to use with $\mathbf{k}$ space methods for solving the EMA equations. In general, we recommend that, before commencing any finite difference calculations of heterostructures, one always examine the finite difference band structures of the constituent bulk materials. This simple procedure can indicate if FDM band structures would lead to spurious solutions.

\section{CONCLUSIONS}

We have analyzed a class of spurious solutions (SSs) for the effective mass approximation (EMA), which appears when solving the EMA equations with the finite difference method (FDM). A general approach to the study of this class of SSs has been formulated, and has been applied to the eight-band EMA. We have shown that looking at the bulk band structure of the constituents along the discretization direction constitutes a very valuable tool to detect potentially troublesome situations. We have derived a set of conditions that predict the appearance of SSs. The proposed theory shows excellent agreement with the numerical values of the spurious energies. Finally, popular tabulations of Luttinger parameters have been examined with the derived conditions to identify those that might be problematic when carrying out FDM eight-band EMA calculations. The table by Lawaetz ${ }^{45}$ is free from danger, but the parameters in the review article by Vurgaftman and co-workers ${ }^{44}$ can lead to FDM SSs for some of the compounds listed.

\section{ACKNOWLEDGMENTS}

The authors would like to thank Darryl Smith for valuable discussions. This work has been supported by the Office of Naval Research under Grant No. N00014-99-1-1006. A part of this work was carried out at the Jet Propulsion Laboratory, California Institute of Technology, and was sponsored by the Defense Research Projects Agency through an agreement with the National Aeronautics and Space Administration.

${ }^{1}$ J. M. Luttinger and W. Kohn, Phys. Rev. 97, 869 (1955).

${ }^{2}$ G. Bastard, Heterojunctions and Semiconductor Superlattices, 2nd ed. (Springer, New York, 1986).
${ }^{3}$ S. R. White and L. J. Sham, Phys. Rev. Lett. 47, 879 (1981).

${ }^{4}$ G. Bastard, Phys. Rev. B 24, 5693 (1981).

${ }^{5}$ G. Bastard, Phys. Rev. B 25, 7584 (1982).

${ }^{6}$ G. D. Sanders and Y. C. Chang, Phys. Rev. B 32, 4282 (1985).

${ }^{7}$ L. C. Andreani, A. Pasquarello, and F. Bassani, Phys. Rev. B 36, 5887 (1987).

${ }^{8}$ P. C. Sercel and K. J. Vahala, Phys. Rev. B 42, 3690 (1990).

${ }^{9}$ G. A. Baraff and D. Gershoni, Phys. Rev. B 43, 4011 (1991).

${ }^{10}$ H. Ando, S. Nojima, and H. Kanbe, J. Appl. Phys. 74, 6383 (1993).

${ }^{11}$ U. Merkt, J. Huser, and M. Wagner, Phys. Rev. B 43, 7320 (1991).

${ }^{12}$ G. T. Einevoll, Phys. Rev. B 45, 3410 (1992).

${ }^{13}$ I. Suemune, IEEE J. Quantum Electron. 27, 1149 (1991).

${ }^{14}$ M. Rosenzweig, M. Mohrle, H. Duser, and H. Venghaus, IEEE J. Quantum Electron. 27, 1804 (1991).

${ }^{15}$ S. L. Chuang, IEEE J. Quantum Electron. 32, 1791 (1996).

${ }^{16}$ H. Schneider, F. Fuchs, B. Dischler, J. D. Ralston, and P. Koidl, Appl. Phys. Lett. 58, 2234 (1991).

${ }^{17}$ P. Man and D. S. Pan, Appl. Phys. Lett. 61, 2799 (1992).

${ }^{18}$ J. J. Shi and E. M. Goldys, IEEE Trans. Electron Devices 46, 83 (1999).

${ }^{19}$ R. Wessel and M. Altarelli, Phys. Rev. B 39, 12802 (1989).

${ }^{20}$ J. R. Soderstrom, E. R. Brown, C. D. Parker, L. J. Mahoney, J. Y. Yao, T. G. Andersson, and T. C. McGill, Appl. Phys. Lett. 58, 275 (1991).

${ }^{21}$ C. Chao and S. L. Chuang, Phys. Rev. B 43, 7027 (1991).

${ }^{22}$ B. Lee, Superlattices Microstruct. 14, 295 (1993).

${ }^{23}$ V. V. Paranjape, Phys. Rev. B 52, 10740 (1995).

${ }^{24}$ M. Schuurmans and G. W. 't Hooft, Phys. Rev. B 31, 8041 (1985).

${ }^{25}$ M. J. Godfrey and A. M. Malik, Phys. Rev. B 53, 16504 (1996).

${ }^{26}$ L. W. Wang, Phys. Rev. B 61, 7241 (2000).

${ }^{27}$ P. C. Sercel, A. L. Efros, and M. Rosen, Phys. Rev. Lett. 83, 2394 (1999).

${ }^{28}$ W. Jaskolski, R. Oszwaldowski, and G. W. Bryant, Vacuum 63, 191 (2001).

${ }^{29}$ R. Eppenga, M. F. H. Schuurmans, and S. Colak, Phys. Rev. B 36, 1554 (1987).

${ }^{30}$ B. A. Foreman, Phys. Rev. B 56, 12748 (1997).

${ }^{31}$ M. G. Burt, Superlattices Microstruct. 23, 531 (1998).

${ }^{32}$ S. Panda, B. K. Panda, S. Fung, and C. D. Beling, J. Appl. Phys. 80, 1532 (1996).

${ }^{33}$ Y. X. Liu, D. Z.-Y. Ting, and T. C. McGill, Phys. Rev. B 54, 5675 (1996).

${ }^{34}$ S. L. Chuang and C. S. Chang, Semicond. Sci. Technol. 12, 252 (1997).

${ }^{35}$ S. F. Tsay, J. C. Chiang, Z. M. Chau, and I. Lo, Phys. Rev. B 56, 13242 (1997).

${ }^{36}$ Q. Fanyao, A. L. A. Fonseca, and O. A. C. Nunes, Superlattices Microstruct. 26, 377 (1999).

${ }^{37}$ O. Stier, M. Grundmann, and D. Bimberg, Phys. Rev. B 59, 5688 (1999).

${ }^{38}$ C. R. Pidgeon and R. N. Brown, Phys. Rev. 146, 575 (1966).

${ }^{39}$ Except, of course, for a very coarse grid, where, as per the Nyquist theorem, there are not enough mesh points to properly describe the oscillation of the envelope function in the confinement region.

${ }^{40}$ Q. G. Zhu and H. Kroemer, Phys. Rev. B 27, 3519 (1983).

${ }^{41}$ D. Z.-Y. Ting, E. T. Yu, and T. C. McGill, Phys. Rev. B 45, 3583 (1992).

${ }^{42}$ G. Dresselhaus, Phys. Rev. 100, 580 (1955).

${ }^{43}$ J. Taylor and V. Tolstikhin, J. Appl. Phys. 87, 1054 (2000).

${ }^{44}$ I. Vurgaftman, J. R. Meyer, and L. R. Ram-Mohan, J. Appl. Phys. 89, 5815 (2001)

${ }^{45}$ P. Lawaetz, Phys. Rev. B 4, 3460 (1971).

${ }^{46}$ S. L. Chuang, Phys. Rev. B 43, 9649 (1991).

${ }^{47}$ O. E. Madelung, Semiconductors—Basic Data, 2nd ed. (Springer, Berlin, 1996). 